\title{
Emergent hydrodynamics in microscopic modeling of early stage of relativistic heavy-ion collisions
}

\author{
L. V. Bravina ${ }^{1,2, a}$, E. E. Zabrodin ${ }^{1,2}$ \\ ${ }^{1}$ Department of Physics, University of Oslo, Oslo, Norway \\ ${ }^{2}$ Skobeltsyn Institute of Nuclear Physics, Lomonosov Moscow State University, Moscow, Russia
}

Received: 15 June 2020 / Accepted: 28 September 2020 / Published online: 6 October 2020

(C) The Author(s) 2020

Communicated by Laura Tolos

\begin{abstract}
Time evolution of hot and dense nuclear matter produced in central gold-gold collisions at energies between $E_{l a b}=10$ and $160 \mathrm{AGeV}$ is studied within two transport string models, UrQMD and QGSM. In contrast to the previous studies, here we investigate the macroscopic characteristics of the system before the state of chemical and thermal equilibrium is attained. For all energies in question two interesting observations are made for times starting already from $t \geq 1 \mathrm{fm} / c$. (1) The matter in the cell expands almost isentropically with nearly constant entropy per baryon. (2) Pressure in the cell appears to be very close to the pressure calculated for equilibrated hadron gas with the same values of energy density, baryon density and strangeness density. The pressure linearly depends on the energy density, $P=a(\sqrt{s}) \varepsilon$. Therefore, both observations endorses the formal application of relativistic hydrodynamics from the very early stages of heavy-ion collisions, despite of the fact that the matter in the fireball is out of equilibrium.
\end{abstract}

\section{Introduction}

Hydrodynamic, or rather hybrid, models are common tools nowadays for the description of heavy-ion and hadronnucleus collisions at relativistic energies, for review see $[1,2]$ and references therein. The original model of hydrodynamic description of multiparticle production in relativistic collisions was formulated by Landau in $[3,4]$. Its basic postulates are as follows. The first stage of the collision consists of interpenetration of two Lorentz-contracted nuclei which results to momentary stopping and formation of a hot blob of violently fluctuating liquid. Pomeranchuk once called it "boiling liquid of operators". The second stage deals with the expansion of hot and dense matter governed by relativistic hydrodynamics.

a e-mail: larissa.bravina@fys.uio.no (corresponding author)
Its main equation reads

$\partial_{\mu} T^{\mu \nu}=0$

where $T^{\mu \nu}$ is the energy-momentum tensor

$T^{\mu \nu}=(\varepsilon+P) u^{\mu} u^{\nu}+P g^{\mu \nu}+\eta^{\mu \nu}$,

containing pressure $P$, energy density $\varepsilon$, local four-velocity $u^{\mu}$, and dissipative tensor $\eta^{\mu \nu}$. In the first approximation one may consider perfect fluid with no dissipative processes. Expansion of such fluid proceeds isentropically. Since $u^{\mu} u_{\mu}=1$, we have five equations and six unknown variables, namely, four components of $u^{\mu}, \varepsilon$, and $P$, meaning that one equation is still missing. The missing equation is the well-known equation of state (EOS) which links pressure to energy density. Landau has picked up the EOS of gas of ultrarelativistic particles,

$P=c_{s}^{2} \varepsilon=\frac{1}{3} \varepsilon$,

where the proportionality coefficient is the square of the speed of sound. Later on the estimate of the sonic speed in multicomponent hadronic gas with resonances was diminished from $c_{s}^{2}=1 / 3$ to $c_{s}^{2} \approx 1 / 5$ [5] and then to $c_{s}^{2} \approx 1 / 7$ [6]. Further consideration of the expanding system takes place under fundamental assumption of local thermodynamic equilibrium. Note, that the initial conditions in Landau model are relativistically non-invariant. This imperfection is fixed in the Bjorken model [7]. The latter postulates that the thermodynamic characteristics of the system are constant on the hypersurface $\tau=$ const, where the proper time is $\tau=\sqrt{t^{2}-z^{2}}$, with $t$ and $z$ being time and coordinate along the beam axis, respectively. In terms of $\tau$ one gets from Eq. (1)

$\frac{d \varepsilon}{d \tau}+\frac{\varepsilon+P}{\tau}=0$ 
Inserting in Eq. (4) thermodynamic relations

$$
\begin{aligned}
\varepsilon+P & =T s \\
d \varepsilon & =T d s
\end{aligned}
$$

containing temperature $T$ and entropy density $s$, we arrive again to the condition of isentropic expansion

$$
\frac{d s}{d \tau}+\frac{s}{\tau}=0
$$

Modern hydrodynamic models usually include hadronic cascade as afterburner after the chemical freeze-out in the system, when the inelastic collisions have ceased. They nicely describe the basic features of heavy-ion collisions in a broad range of bombarding energies $[8,9]$. Nevertheless, all models employ the assumption of local thermal equilibrium in the expanding fireball. It appears that the thermalization time needed to match the experimental data is very short, e.g. $\tau \approx 0.1 \div 0.5 \mathrm{fm} / c$. Such an extremely rapid thermalization looks a bit suspicious. Several possible explanations have been proposed. For instance, the approach formulated in [10] concludes that hydrodynamic description with a large coarse-graining scale is sufficient for observables which do not require a precise space-time resolution of the system. In this case, the local thermal equilibrium is not a necessary prerequisite of the hydrodynamic description. According to other explanation, the low-order hydrodynamics can well approximate the hydrodynamic attractor solution describing out-of-equilibrium systems, thus justifying its applicability $[11,12]$. Hydrodynamization of nuclear matter produced in relativistic heavy-ion collisions has been studied extensively in the last years, see, e.g., [13-15] and references therein. One of the interesting results states [13] that the hydrodynamization time is proportional to shear viscosity $\eta$ and inversely proportional to entropy density $s$ and temperature $T$

$\tau_{\text {hydro }} \sim \frac{\eta / s}{T}$

Note, however, that rigorous definition of temperature does not exist for the out-of-equilibrium system.

There is another group of models, which do not based on the assumption of local equilibrium, namely, microscopic transport models. Relaxation of hot and dense matter, produced in the central area of relativistic heavy-ion collisions, to local equilibrium in microscopic model calculations was studied in [16-26]. In present paper the employed two different transport models are ultra-relativistic quantum molecular dynamics (UrQMD) [27,28] and quark-gluon string model (QGSM) [29,30]. Results of microscopic calculations are compared to those of statistical model (SM) of ideal hadron gas with essentially the same degrees of freedom. In previous studies it was found that at any collision energy the system nearly attains the state of chemical and thermal equilibrium irrespective of the model employed. However, relaxation pro- cess needs at least $t=6 \mathrm{fm} / c$ or longer. The main goal of our paper is to investigate the fulfillment of basic conditions for formal application of hydrodynamics before the equilibrium is reached.

The paper is organized as follows. Description of similarities and differences between the two microscopic transport models is given in Sect. 2. Section 3 presents basic features of the statistical model (SM) of ideal hadron gas. Study of the evolution of bulk characteristics of matter produced in the central area of central heavy-ion collisions is performed in Sect. 4. Energies of colliding nuclei are varying from $E_{\text {lab }}=10$ to $160 \mathrm{AGeV}$ accessible for both NICA and FAIR facilities (up to approximately $40 \mathrm{AGeV}$ ) and for SPS CERN (up to $160 \mathrm{AGeV}$ ). Of primary interest is the evolution of energy density, pressure gradients, entropy per baryon, as well as comparison of these parameters with those calculated for the ideal hadron gas in chemical and thermal equilibrium. Conclusions are drawn in Sect. 5.

\section{Similarities and differences between the microscopic models}

Both UrQMD [27,28] and QGSM [29,30] are designed as Monte Carlo event generators for the description of hadron-hadron $(h h)$, hadron-nucleus $(h A)$ and nucleus-nucleus $(\mathrm{A}+\mathrm{A})$ collisions in a very broad range of bombarding energies. Particles propagate according to Hamilton equations of motion. They can interact both elastically and inelastically if the distance $d$ between their centers is smaller than $\sqrt{\left(\sigma^{\text {tot }} / \pi\right)}$, where $\sigma^{\text {tot }}$ is the total cross section of the interaction. This is the so-called "black disk" approximation. Production of new particles takes place via the excitation and fragmentation of colored strings. The UrQMD applies the classical Lund picture [31] of the string longitudinal excitation. Here the strings are stretching between the (anti)quarks and (anti)diquarks, belonging to the same hadron. The string masses arise due to the momentum transfer, and the string always splits into a substring and a hadron on a mass shell. For hard processes with the momentum transfer larger than $1.5 \mathrm{GeV} / c$ UrQMD uses PYTHIA [32]. In contrast, the QGSM belongs to the class of models based on the Gribov Reggeon field theory $[33,34]$. In these models the strings are stretching between the constituents belonging to different hadrons, and the string masses emerge due to the color exchange mechanism. The number of various subprocesses is very rich and the method is known as $1 / N$ or topological expansion $[35,36]$ of Lagrangian in quantum chromodynamics (QCD). Although it is not possible to assign a proper weight to each arising diagram within the first-principles theory, these diagrams appear to have one-to-one correspondence to the processes going via exchange of certain amount of Pomerons and Reggeons in the RFT [37-39]. 
Decays of strings in the QGSM proceed according to the Field-Feynman algorithm [40] with independent hadron formation from both ends of the string. Similarly for both models, strings are stretching uniformly between the constituents with the constant string tension $\kappa \approx 1 \mathrm{GeV} / \mathrm{fm}$. Production of new hadrons proceeds via the Schwinger-like mechanism of quark-antiquark and diquark-antidiquark pair creation. The produced particles are uniformly distributed in the rapidity space. Both models employ the available experimental data, such as cross sections of hadrons, widths of resonances and their decay modes. In case of lacking the information, the models apply the one-boson exchange model, isospin symmetry conditions, and detailed balance consideration. Tables of resonances in both models, however, are different. For the description of $h A$ and $\mathrm{A}+\mathrm{A}$ collisions hadronic rescattering scheme, or hadronic cascade, is implemented. Because of the uncertainty principle, newly produced hadrons cannot interact immediately. They become on-shell particles only after a certain formation time, see $[42,43]$ and references therein. Only the hadrons containing the valence quarks can interact immediately after the rescattering with the reduced interaction cross section. The Pauli principle, prohibiting the creation of a fermion if the outgoing phase space is already occupied, is implemented, whereas no Bose enhancement effects are taken into account. Further details of the models can be found elsewhere [27-30,41,44]. It was our intention to choose two models which have quite different underlying principles. In this case one may expect significant reduction of the influence of model-dependent effects on the analysis of relaxation of hot and dense partonic matter in heavy-ion collisions in a broad energy range.

\section{Basic principles of statistical model}

If the system of hadrons reaches the state of thermal and chemical equilibrium, its properties are fully determined by the distribution functions of hadron species. In system of natural units with $c=k_{B}=\hbar=1$, the distribution function for a hadronic specie " $i$ " at temperature $T$ reads

$f\left(p, m_{i}\right)=\left[\exp \left(\frac{E_{i}-\mu_{i}}{T}\right) \pm 1\right]^{-1}$

Here $p$ is the full momentum, $m_{i}$ is the mass, $E_{i}$ is the energy, $E_{i}^{2}=p^{2}+m_{i}^{2}$, and $\mu_{i}$ is the total chemical potential of the $i$-th hadron specie, respectively. The "+" sign is for Fermi-Dirac statistics, and the "-" sign stands for BoseEinstein one. Obviously, the $\mu_{i}$ depends on the chemical potentials associated with the conserved charges in strong interactions, i.e. baryon charge $B_{i}$ and strangeness content $S_{i}$ of the hadron,

$\mu_{i}=B_{i} \mu_{B}+S_{i} \mu_{S}$, where $\mu_{B}$ and $\mu_{S}$ are the baryon- and the strangeness chemical potentials. ${ }^{1}$

Hadron number density and energy density of the $i$ th hadron specie with spin-isospin degeneracy $g_{i}$ are the first and the second moments of the distribution function $f\left(p, m_{i}\right)$, respectively

$\begin{aligned} n_{i} & =\frac{g_{i}}{(2 \pi)^{3}} \int f\left(p, m_{i}\right) d^{3} p, \\ \varepsilon_{i} & =\frac{g_{i}}{(2 \pi)^{3}} \int E_{i} f\left(p, m_{i}\right) d^{3} p .\end{aligned}$

The partial hadron pressure is given by

$P_{i}=\frac{g_{i}}{(2 \pi)^{3}} \int \frac{p^{2}}{3 E_{i}} f\left(p, m_{i}\right) d^{3} p$,

and the entropy density reads

$s=-\sum_{i} \frac{g_{i}}{(2 \pi)^{3}} \int f\left(p, m_{i}\right)\left[\ln f\left(p, m_{i}\right)-1\right] d^{3} p$.

We see that all thermodynamic characteristics of the system are functions of three parameters, $T, \mu_{B}$ and $\mu_{S}$. In order to find these parameters one has to extract energy density $\varepsilon^{m i c}$, baryon density $\rho_{B}^{m i c}$ and strangeness density $\rho_{S}^{m i c}$ from the selected volume in microscopic calculations and insert it into a system of equations

$$
\begin{aligned}
\varepsilon^{m i c} & =\sum_{i} \varepsilon_{i}\left(T, \mu_{B}, \mu_{S}\right), \\
\rho_{B}^{m i c} & =\sum_{i} B_{i} n_{i}\left(T, \mu_{B}, \mu_{S}\right), \\
\rho_{S}^{m i c} & =\sum_{i} S_{i} n_{i}\left(T, \mu_{B}, \mu_{S}\right) .
\end{aligned}
$$

The number of particle states employed as independent degrees of freedom in microscopic and macroscopic calculations should be essentially the same. If the system of hadrons in microscopic model calculations is in the vicinity of equilibrium, its partial particle abundances and particle energy spectra have to be close to those given by the statistical model. Note, that we do not use for the comparison the statistical model with the excluded volume effects, see e.g. $[45,46]$. This is done because the system of hadrons in microscopic model resembles an ideal gas of point-like particles rather than finite-volume ones, despite of the fact that the average interaction distance between the hadrons is far from zero, see [47] for details.

\footnotetext{
${ }^{1}$ We drop the dependence on electric charge or isospin chemical potential, because their values are order of magnitude weaker compared to $\mu_{B}$ and $\mu_{S}$.
} 


\section{Early stage of heavy-ion collisions}

Similar to the previous analysis, we opted for the cubic cell with volume $V=5 \times 5 \times 5=125 \mathrm{fm}^{3}$ centered around the center-of-mass of colliding heavy ions. Obviously, at the very beginning of the collision the pressure gradients along the beam axis $z$ and in the transverse plane $(x, y)$ must be different. One can calculate the components of the pressure tensor $P_{j}, j=\{x, y, z\}$ by using the virial theorem [48]

$P_{j}=\frac{1}{3 V} \sum_{i} \frac{p_{i\{j\}}^{2}}{E_{i}}$,

where $V$ is the volume of the cell, $p_{i\{j\}}$ is the $j$-component of the momentum of $i$-th hadron, and $E_{i}=\left(m_{i}^{2}+p_{i}^{2}\right)^{1 / 2}$ is its total energy. After that the total pressure $P^{t o t}$ in the system can be calculated. Because of the radial symmetry of $A+A$ system of nuclei colliding with zero impact parameter, it is enough to compare just two tripled diagonal components of the pressure tensor, $3 P_{x}$ and $3 P_{z}$, with $P^{\text {tot }}$. Results of the

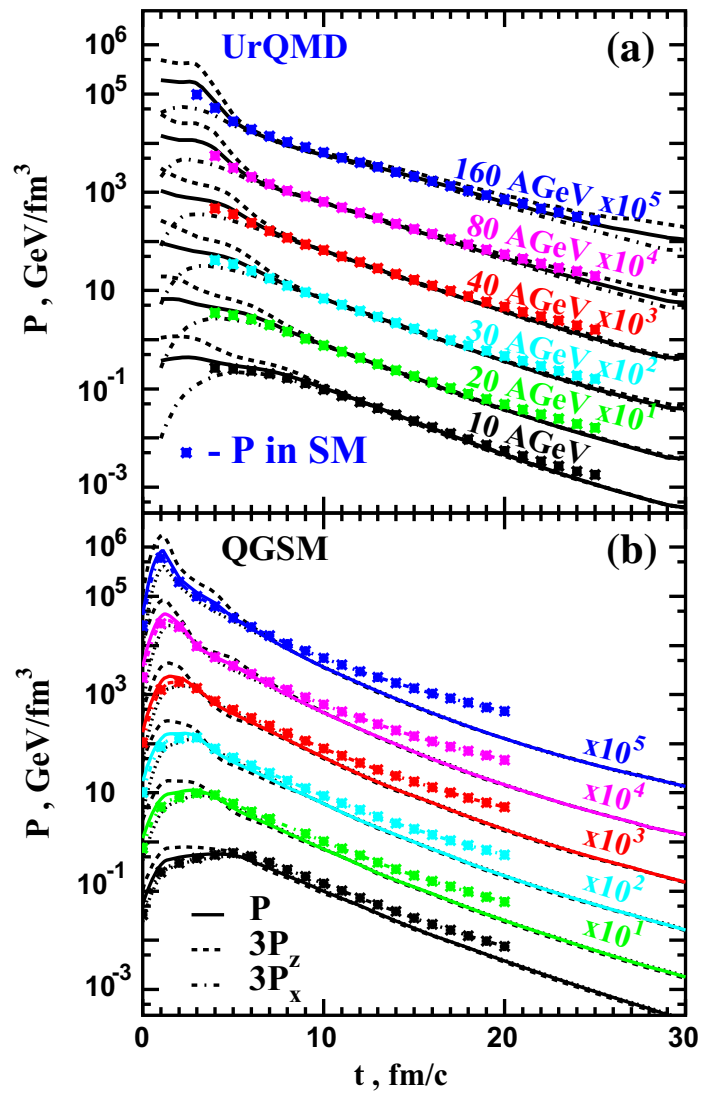

Fig. 1 (Color online) The tripled transverse ( $3 P_{x}$, dash-dotted curves) and the tripled longitudinal ( $3 P_{z}$, dashed curves) diagonal components of the microscopic pressure tensor in the central cell with volume $V=125 \mathrm{fm}^{3}$ in $\mathbf{a}$ UrQMD and $\mathbf{b}$ QGSM calculations of central $\mathrm{Au}+\mathrm{Au}$ collisions at energies from $E_{l a b}=10$ to $160 \mathrm{AGeV}$. Solid curves indicate the total microscopic pressure, and asterisks denote the pressure given by the statistical model microscopic calculations are shown in Fig. 1. The statistical model pressure, calculated according to Eq. (13), is plotted onto the microscopic results also. We see that for all four bombarding energies the system quickly develops the transverse pressure. Convergence of $P_{z}$ and $P_{T}$, however, takes place at $t=7-8 \mathrm{fm} / c$ in the UrQMD calculations and at $t=4-6 \mathrm{fm} / c$ in the QGSM ones. Thus, there is no even kinetic equilibrium, which assumes isotropy of the pressure, in the system at early times, not to speak about chemical or thermal equilibrium. These times can be diminished somehow. As was shown in [26], the disregard of spectators and application of less strict isotropization criterion within the same UrQMD model can decrease the isotropization time of pressure to $t=3-6 \mathrm{fm} / c$. And transition to macroscopic hydrodynamic evolution can occur even for rather anisotropic systems with $P_{T} / P_{z} \sim 0.5[13,15]$. Thus, the beginning of hydrodynamic description may take place significantly earlier compared to rather conservative numbers extracted from Fig. 1. What surprises us further is that the pressure $P^{S M}$, obtained for the hadron gas under complete thermal and chemical equilibrium, appears to be very close to the total microscopic pressure in the central cell even at $t \approx 1 \mathrm{fm} / c$ after beginning of the collision. Note, that the cell is an open system. Its particle composition is rapidly changing during the fireball expansion, whereas the energy density drops. However, one can study the time evolution of the entropy-per-baryon ratio, $s / \rho_{B}$. This ratio is displayed in Fig. 2 for $\mathrm{Au}+\mathrm{Au}$ collisions at all six energies in question. For all reactions entropy-per baryon seems to be quite stable within the $10 \%$ accuracy limit for both employed models. At early stage of the collision, $t \leq 2 \mathrm{fm} / c$, the ratio $s / \rho_{B}$ is just a bit larger than that at $t \geq 3 \mathrm{fm} / c$. Note also that the entropy density here is overestimated for early stages of the collisions, because the entropy density in non-equilibrium stage is lower than the equilibrium entropy density. In case of microscopically determined entropy density the ratio $s^{m i c} / \rho_{B}$ becomes rather flat, see [21]. One may conclude that the expansion proceeds nearly isentropically with the almost constant entropyper-baryon ratio. This behavior is similar to the isentropic expansion of ideal hadronic fluid.

Now we can study the simultaneous evolution of the pressure and the energy density in the cell. Although the microscopic and the macroscopic pressures appear to be very close to each other, as shown in Fig. 1, we plot the evolution of $P^{m i c}(\varepsilon)$ and $P^{S M}(\varepsilon)$ separately in Fig. 3 and in Fig. 4, respectively. Macroscopic pressure $P^{S M}$ demonstrates remarkably linear dependence on the energy density, $P^{S M}=a \varepsilon, a \approx$ const from the very beginning of the collision. The slope, which is related to the speed of sound in the system as $a=c_{s}^{2}$, remains nearly constant throughout the expansion of the system. For microscopic pressure $P^{m i c}$ some deviations from the straight line behavior are seen, i.e. the slopes $d P / d \varepsilon$ of the distributions are steeper at earlier 


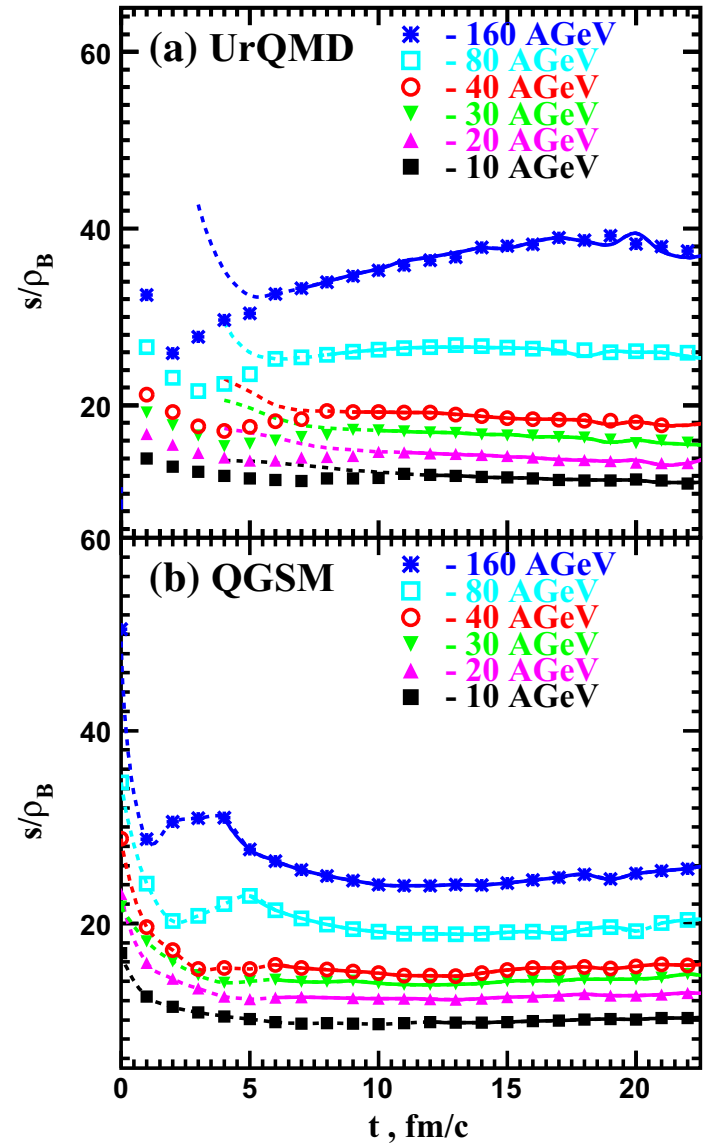

Fig. 2 (Color online) Time evolution of the entropy-per-baryon ratio $s / \rho_{B}$ in the central cell with volume $V=125 \mathrm{fm}^{3}$ in a UrQMD and b QGSM calculations of central $\mathrm{Au}+\mathrm{Au}$ collisions at energies from $E_{l a b}=10$ to $160 \mathrm{AGeV}$, respectively. Dashed lines denote the nonequilibrium stage of the collision, whereas solid lines indicate the equilibrium phase

times. Also, the slopes are slightly increasing with rising collision energy. This behavior emulates the hydrodynamic expansion of nuclear matter, though the conditions of local chemical and thermal equilibrium are not fulfilled yet. The slope parameter varies from $a=0.130(0.125)$ at $E_{l a b}=10$ to $0.145(0.138)$ at $E_{l a b}=160 \mathrm{AGeV}$ in UrQMD (QGSM) calculations. These numbers are close to those obtained in [49] within the UrQMD hybrid approach, but the latters rise steeper with increasing bombarding energy. Possible explanation is that we study just the central cell and not the whole rapidity region as in [49]. It is quite interesting also that the best agreement with measured mean transverse mass distribution at energies below $\sqrt{s}=10 \mathrm{GeV}$ is obtained in [49] for calculations with a hadron resonance gas equation of state with $c_{s}^{2}=1 / 8=0.125$, which matches our data very well.

To see the possible deviations of bulk characteristics of the system from the ideal gas ones, it is instructive to check the evolution of the energy density and the entropy density with the temperature in microscopic model calculations. Evo-

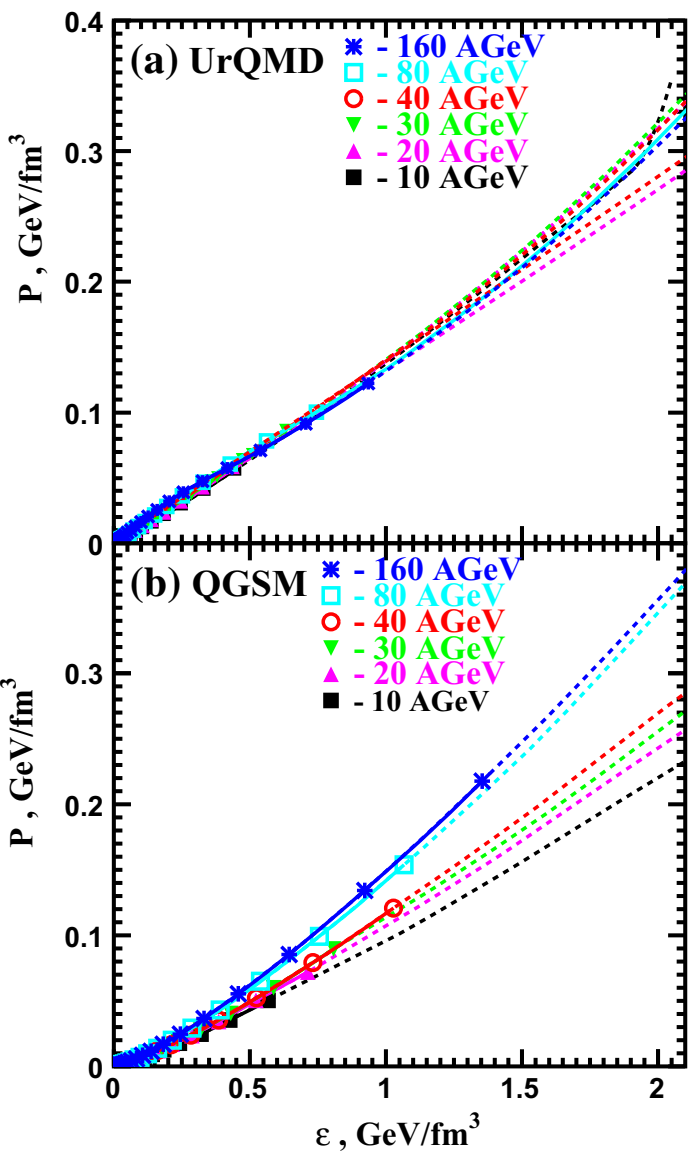

Fig. 3 (Color online) Time evolution of the microscopic pressure $P^{m i c}$ and the energy density $\varepsilon$ in the central cell with volume $V=125 \mathrm{fm}^{3}$ in $\mathbf{a}$ UrQMD and $\mathbf{b}$ QGSM calculations of central Au+Au collisions at energies from $E_{l a b}=10$ to $160 \mathrm{AGeV}$, respectively. Dashed lines denote the non-equilibrium stage of the collision, whereas solid lines indicate the equilibrium phase

lutions of these parameters as functions of temperature $T$ within the expanding perfect fluid with zero chemical potential are related to the speed of sound as follows. Combining the equations

$d P=s d T$,

$d P=a d \varepsilon$,

with Eqs. (5) and (6), one gets

$$
\begin{aligned}
\frac{d \varepsilon}{\varepsilon} & =\frac{1+a}{a} \frac{d T}{T}, \\
\frac{d s}{s} & =\frac{1}{a} \frac{d T}{T} .
\end{aligned}
$$

Integration of the last two equations provides us the desired dependencies

$$
\frac{\varepsilon}{\varepsilon_{0}}=\left(\frac{T}{T_{0}}\right)^{\frac{1+a}{a}},
$$




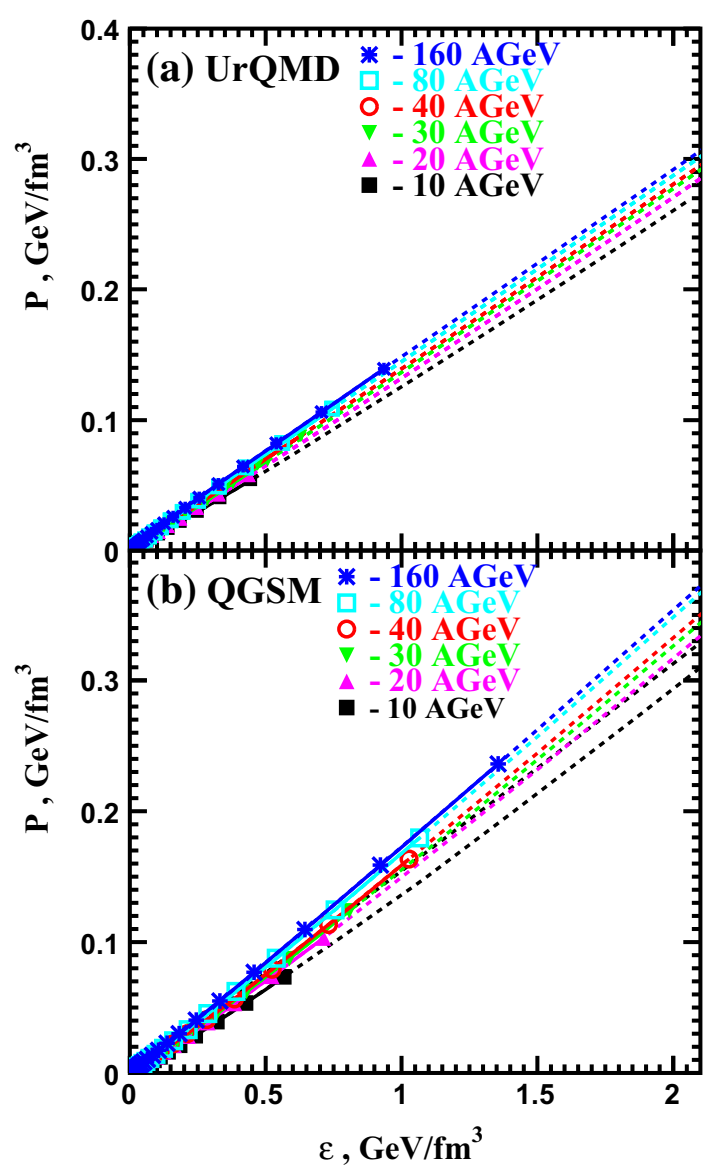

Fig. 4 (Color online) The same as Fig. 3 but for macroscopic pressure $P^{S M}$ defined from the $\mathrm{SM}$ fit to extracted microscopic parameters $\varepsilon, \rho_{B}, \rho_{S}$

$$
\frac{s}{s_{0}}=\left(\frac{T}{T_{0}}\right)^{\frac{1}{a}}
$$

Figure 5 displays the evolution of ratios $\varepsilon / \varepsilon_{0}$ vs $T / T_{0}$ (Fig. 5a) and $s / s_{0}$ vs $T / T_{0}$ (Fig. 5b) calculated in the central cell for two energies, $E_{l a b}=20$ and $40 \mathrm{AGeV}$. One can see that the difference between the model predictions for these energies is very small. Calculations for ideal hadron gas made according to Eqs. (23) and (24) with $a=0.14$ are plotted onto the model results as well. The difference between the curves is weak indicating that effects caused by deviations from equilibrium, and non-zero baryon and strangeness chemical potentials do not play a decisive role here. The fit of the microscopic distributions to both Eqs. (23) and (24) provides us exactly $a=c_{s}^{2}=0.2$. In other words, behavior of initially non-equilibrated hot and dense matter in transport model calculations can be approximated by hydrodynamic model of nearly perfect fluid almost from the very beginning of nuclear collision.

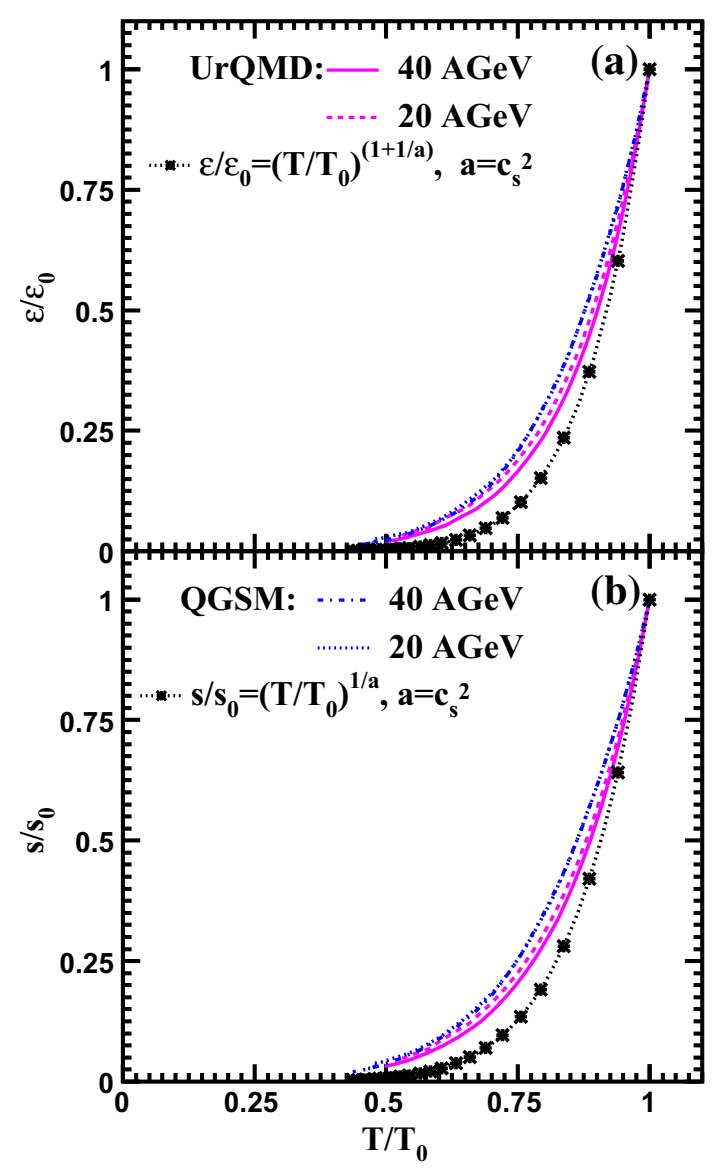

Fig. 5 (Color online) a Evolution of energy densities $\varepsilon / \varepsilon_{0}$ with temperature $T / T_{0}$ in the central cell with volume $V=125 \mathrm{fm}^{3}$ in central $\mathrm{Au}+\mathrm{Au}$ collisions. Solid line and dashed line denote UrQMD calculations at $E_{l a b}=40$ and $E_{l a b}=20 \mathrm{AGeV}$, respectively. Dash-dotted line and dotted line represent the QGSM results for these reactions. Analytic calculations given by Eq. (23) with $a=0.14$ are shown by asterisks. $\mathbf{b}$ The same as for a but for the evolution of entropy densities $s / s_{0}$ with temperature $T / T_{0}$. Asterisks show the calculations according to Eq. (24)

\section{Conclusions}

We have studied the evolution of energy density, entropy density and pressure gradients in the central cubic cell with volume $V=5 \times 5 \times 5=125 \mathrm{fm}^{3}$ of central goldgold collisions modeled within the transport models UrQMD and QGSM at bombarding energies from $E_{l a b}=10$ to $E_{l a b}=160 \mathrm{AGeV}$. The models employ different mechanisms of string excitation and fragmentation, and different sets baryon and meson resonances. These circumstances enable us to estimate the range of distortions caused by model-dependent effects. The time step was chosen as $\Delta t=1 \mathrm{fm} / c$. At each time $t=n \Delta t, n=1,2,3, \ldots$ the energy density $\varepsilon$, the net baryon density $\rho_{B}$, and the net strangeness density $\rho_{S}$ were extracted from the cen- 
tral cell in microscopic calculations. These three parameters were inserted then in a set of non-linear equations provided by the statistical model of ideal hadron gas containing essentially the same set of hadronic states as the transport model.

Results of microscopic model calculations show that the extracted total pressure can be well approximated by a linear dependence $P=a \varepsilon$. Parameter $a$, which is the square of the sonic velocity in the system, $a=c_{s}^{2}$, is slightly decreasing as the system becomes more dilute. Also $a$ increases with increasing bombarding energy from $0.13(0.125)$ at $E_{l a b}=10 \mathrm{GeV}$ to $0.145(0.138)$ at $E_{l a b}=160 \mathrm{GeV}$ in the UrQMD(QGSM) calculations. This ratio becomes nearly constant almost from the very early stage of nuclear collisions, at $t \geq 1 \mathrm{fm} / c$. Then, the hot and dense nuclear matter expands practically isentropically with the conservation of the entropy per baryon. Both conditions are necessary prerequisites for application of hydrodynamic description. But, in presence of large pressure anisotropy the microscopic energy-momentum tensor cannot be mapped to the energy-momentum tensor of ideal, or viscous, hydrodynamics. On the other hand, pressure gradients in longitudinal and transverse directions are rapidly equalized. This means that the hydrodynamic stage can come before the conditions of local thermal and chemical equilibrium are fulfilled (typically, about $6-10 \mathrm{fm} / \mathrm{c}$ after beginning of heavy-ion collisions).

Acknowledgements Fruitful discussions with M. Bleicher, K. Bugaev, L. Csernai, Yu. Ivanov and H. Stöcker are gratefully acknowledged. This work was supported by Russian Foundation for Basic Research (RFFI) under Grant No. 18-02-40084 and Grant No. 18-02-40085. The authors are grateful to the COST Action CA15213 "THOR" for supporting their networking.

Funding Open Access funding provided by University of Oslo (incl Oslo University Hospital).

Data Availability Statement This manuscript has no associated data or the data will not be deposited. [Authors' comment: We do not store generation data. Simulation results obtained using the UrQMD can be easily reproduced since the model is freely available.]

Open Access This article is licensed under a Creative Commons Attribution 4.0 International License, which permits use, sharing, adaptation, distribution and reproduction in any medium or format, as long as you give appropriate credit to the original author(s) and the source, provide a link to the Creative Commons licence, and indicate if changes were made. The images or other third party material in this article are included in the article's Creative Commons licence, unless indicated otherwise in a credit line to the material. If material is not included in the article's Creative Commons licence and your intended use is not permitted by statutory regulation or exceeds the permitted use, you will need to obtain permission directly from the copyright holder. To view a copy of this licence, visit http://creativecomm ons.org/licenses/by/4.0/.

\section{References}

1. C. Gale, S. Jeon, B. Schenke, Int. J. Mod. Phys. A 28, 1340011 (2013)

2. P. Huovinen, P.V. Ruuskanen, Ann. Rev. Nucl. Part. Sci. 56, 163 (2006)

3. L.D. Landau, Izv. Akad. Nauk SSSR Ser. Fiz. 17, 51 (1953). (in Russian)

4. S.Z. Belenkij, L.D. Landau, Suppl. Nuovo Cim. 3, 15 (1956)

5. E. Shuryak, Sov. J. Nucl. Phys. 16, 395 (1972)

6. J. Letessier, J. Rafelski, Hadrons and Quark-Gluon Plasma (Cambridge University Press, Cambridge, 2002)

7. J.D. Bjorken, Phys. Rev. D 27, 140 (1983)

8. Proc. Quark Matter 2017, ed. by U. Heinz, O. Evdokimov, P. Jacobs, Nucl. Phys. A 967, 1-1010 (2017)

9. Proc. Quark Matter 2018, ed. by F. Antinori, A. Dainese, P. Giubellino, V. Greco, M.P. Lombardo, E. Scomparin, Nucl. Phys. A 982, 1-1066 (2019)

10. Ph Mota, T. Kodama, R.D. de Souza, J. Takahashi, Eur. Phys. J. A 48, $165(2012)$

11. M.P. Heller, M. Spalinski, Phys. Rev. Lett. 115, 072501 (2015)

12. P. Romatschke, Eur. Phys. J. C 77, 21 (2017)

13. M.P. Heller, A. Kurkela, M. Spalinski, V. Svensson, Phys. Rev. D 97, 091503 (2018)

14. A. Kurkela, A. Mazeliauskas, J.-F. Paquet, S. Schlichting, D. Teaney, Phys. Rev. Lett. 122, 122302 (2019)

15. A. Mazeliauskas, Nucl. Phys. A 982, 134 (2019)

16. L.V. Bravina et al., Phys. Lett. B 434, 379 (1998)

17. L. Bravina et al., J. Phys. G 25, 351 (1999)

18. L. Bravina et al., Phys. Rev. C 60, 024904 (1999)

19. L.V. Bravina, E.E. Zabrodin, S.A. Bass, M. Bleicher, M. Brandstetter, S. Soff, H. Stöcker, W. Greiner, Phys. Rev. C 62, 064906 (2000)

20. E.L. Bratkovskaya, W. Cassing, C. Greiner, M. Effenberger, U. Mosel, A. Sibirtsev, Nucl. Phys. A 675, 661 (2000)

21. L.V. Bravina, E.E. Zabrodin, S.A. Bass, M. Bleicher, M. Brandstetter, Amand Faessler, C. Fuchs, W. Greiner, S. Soff, H. Stöcker, Phys. Rev. C 63, 064902 (2001)

22. L.V. Bravina, E.E. Zabrodin, S.A. Bass, Amand Faessler, C. Fuchs, M.I. Gorenstein, W. Greiner, S. Soff, H. Stöcker, H. Weber, Nucl. Phys. A 698, 383c (2002)

23. L. Bravina, I. Arsene, M.S. Nilsson, K. Tywoniuk, E. Zabrodin, M. Bleicher, H. Stöcker, W. Greiner, Int. J. Mod. Phys. E 16, 777 (2007)

24. L.V. Bravina et al., Phys. Rev. C 78, 014907 (2008)

25. E.E. Zabrodin et al., J. Phys. G 36, 064065 (2009)

26. D. Oliinychenko, H. Petersen, Phys. Rev. C 93, 034905 (2016)

27. S.A. Bass et al., Prog. Part. Nucl. Phys. 41, 255 (1998)

28. M. Bleicher et al., J. Phys. G 25, 1859 (1999)

29. N.S. Amelin, L.V. Bravina, Sov. J. Nucl. Phys. 51, 133 (1990)

30. N.S. Amelin, L.V. Bravina, L.P. Csernai, V.D. Toneev, K.K. Gudima, SYu. Sivoklokov, Phys. Rev. C 47, 2299 (1993)

31. B. Andersson, G. Gustafson, B. Nielsson-Almqvist, Nucl. Phys. B 281, 289 (1987)

32. T. Sjostrand, S. Mrenna, P. Skands, Comput. Phys. Commun. 178, 852 (2008)

33. V. Gribov, Sov. Phys. JETP 26, 414 (1968)

34. L.V. Gribov, E.M. Levin, M.G. Ryskin, Phys. Rep. 100, 1 (1983)

35. G. t'Hooft, Nucl. Phys. B 75, 461 (1974)

36. G. Veneziano, Phys. Lett. B 52, 220 (1974)

37. A.B. Kaidalov, Phys. Lett. B 116, 459 (1982)

38. A.B. Kaidalov, K.A. Ter-Martirosyan, Phys. Lett. B 117, 247 (1982) 
39. A.B. Kaidalov, Surv. High Energy Phys. 13, 265 (1999)

40. R.D. Field, R.P. Feynman, Nucl. Phys. B 136, 1 (1978)

41. H. Petersen, J. Steinheimer, G. Burau, M. Bleicher, H. Stöcker, Phys. Rev. C 78, 044901 (2008)

42. N.S. Amelin, L.V. Bravina, V.L. Korotkikh, L.I. Sarycheva, SYu. Sivoklokov, Sov. J. Nucl. Phys. 55, 1414 (1992)

43. M.S. Nilsson, L.V. Bravina, E.E. Zabrodin, L.V. Malinina, J. Bleibel, Phys. Rev. D 84, 054006 (2011)

44. J. Bleibel, L.V. Bravina, E.E. Zabrodin, Phys. Rev. D 93, 114012 (2016)
45. D.H. Rischke, M.I. Gorenstein, H. Stoecker, W. Greiner, Z. Phys. C 51, 485 (1991)

46. K.A. Bugaev, Nucl. Phys. A 807, 251 (2008)

47. A. Motornenko, L. Bravina, M.I. Gorenstein, A.G. Magner, E. Zabrodin, J. Phys. G 45, 035101 (2018)

48. M. Berenguer, C. Hartnack, G. Peilert, H. Stöcker, W. Greiner, J. Aichelin, A. Rosenhauer, J. Phys. G 18, 655 (1992)

49. J. Steinheimer, M. Bleicher, Eur. Phys. J. A 48, 100 (2012) 